\title{
Nanosponges: A Novel Drug Delivery System
}

\author{
Ashish Y. Pawar, Apurva K. Naik, Khanderao R. Jadhav \\ Department of Pharmaceutics, Mahatma Gandhi Vidyamandir's Pharmacy College, Panchavati, Nashik, \\ Maharashtra, India
}

\begin{abstract}
The invention of nanosponges (NSs) has become a significant step toward overcoming the problems associated with conventional drug delivery. NS drug delivery system has emerged as one of the most promising fields in life science. These small sponges circulate around the body until they encounter the target site and stick on the surface and began to release the drug in a controlled and predictable manner which is more effective for a particular given dosage. The development of new colloidal carrier called NSs has the potential to solve these problems. Both lipophilic and hydrophilic drugs can be loaded in NSs. NS is a novel and emerging technology which offers controlled drug delivery for topical use. They can be crafted for targeting drugs to a specific site, prevent drug and protein degradation, and prolong the drug release in a controlled manner. NSs play a key role in targeting drug delivery in a controlled manner. In this review article, application of NSs, its preparation methods, and evaluation have been discussed.
\end{abstract}

Key words: Controlled drug delivery, nanosponge, polymers, targeted drug delivery, topical application

\section{INTRODUCTION}

$\mathrm{N}$ anosponges (NSs) are an important part to control the rate of delivery of active agent to the predetermined site by small size and efficient carrier characteristics. NSs are nonmutagenic, nonallergenic, nonirritant, and nontoxic. This is used now in cosmetics over the counter and in sunscreen and also in the prescription product. NSs are porous spheres have a myriad interconnected voids. These NSs have a high capacity of entrapping wide ranges of active ingredients such emollients, fragrances, essential oils, sunscreens, used as a topical carrier system. NSs are prepared by the suitable cross-linking process and also by different organic as well as inorganic materials. NSs can encapsulate various types of the molecules by forming inclusion and noninclusion [Figure 1]. ${ }^{[1]}$

\section{CROSS-LINKING PROCESS}

The cyclodextrin, which is highly crosslinked and highly cross-linked polystyrene (natural derivative of starch), are used for the fabrication of NSs which are insoluble in water and the most common organic solvents, nontoxic, porous, stable above $300^{\circ} \mathrm{C}$ which may be used to encapsulate, carry, and selectively release a great variety of the substances. ${ }^{[2]}$

\section{ORGANIC AND INORGANIC MATERIALS}

Examples of NSs formed using organic and inorganic materials are such as: Titanium or other metal oxide-based NSs, silicon NS particles and those which are carbon-coated metallic NSs. ${ }^{[2]}$

\section{INTERESTING FEATURES OF NSs}

The necessary character of these sponges is their aqueous solubility; this character allows the use of these systems effectively for drugs which have poor solubility. ${ }^{[2]}$ The NSs

\section{Address for correspondence: \\ Dr. Ashish Y. Pawar, Department of Pharmaceutics, Mahatma Gandhi Vidyamandir's Pharmacy College, Panchavati, Nashik - 422 003, Maharashtra, India. \\ Phone: +91-9823481646, \\ E-mail: pawarashish23@gmail.com}

Received: 17-07-2016

Revised: 01-08-2016

Accepted: 11-08-2016 


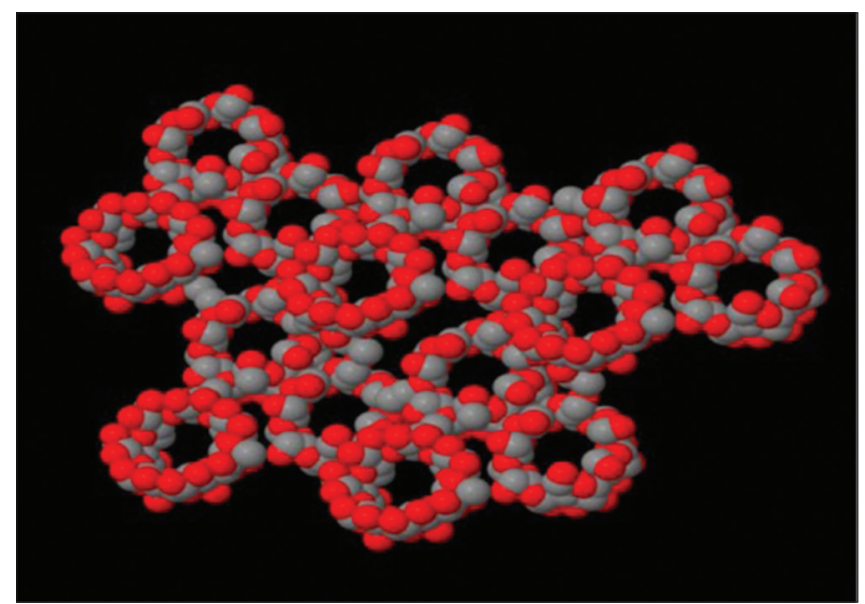

Figure 1: Inclusion formed by nanosponges

are capable to carry both lipophilic and hydrophilic drugs. They are used to increase aqueous solubility of less watersoluble drugs, to remove pollutants from contaminated water, or as nanocarriers for biomedical applications. NSs are used to remove organic impurities in water. ${ }^{[3,4]}$ This technique offers entrapment of the ingredients and reduced the side effects, improved stability, increased elegance, and also enhanced formulation flexibility. Extended releasecontinuous release up to $12 \mathrm{~h}$, it allows incorporation of immiscible liquid for improving material processing-liquid can also be converted to powders. They can be formed in a spherical submicrons particle. NSs can be obtained in a wide range of the dimensions, from 1 to $10 \mu$. The cavities of the framework have a tunable polarity. When using NS as drug delivery system, higher therapeutic activities are observed being the concentration of the active molecule the same. ${ }^{[5,6]}$

The researchers of Vanderbilt University and Emory University has recently reported on a controlled release nanoparticle drug delivery system, which may be improved delivery method for delivering anticancer therapies, including direct injection in the tumor site. These nanoparticles circulate into the body until they encounter the surface of the tumor cell, where they adhere to the surface and start to release the drug in a controlled and predictable manner. ${ }^{[7]}$

The targeted drug delivery systems of this type have several basic advantages. As the drug is released at the tumor site instead of circulating widely through the body, it should be more effective for a given dosage form. They should also have a few harmful adverse effects because smaller amounts of the drug come into contact with the healthy tissue. Another advantage is that the NS particles are also soluble in water. Encapsulating anticancer drug in NS allows the use of hydrophobic drugs that do not dissolve readily in water. These types of drugs should be mixed with adjuvant reagents, which potentially can reduce the efficacy of drug or cause adverse effects. ${ }^{[7,8]}$

\section{PREPARATION OF NANOSPONGES[4,7]}

NSs are prepared using following methods:

\section{Hyper cross-linked - (cyclodextrins)}

Hyper cross-linked cyclodextrin polymers nanostructured to form the three-dimensional networks; a roughly spherical structure, about the size of the protein, with channels and it have pores inside. They are obtained by the reaction of cyclodextrin with a cross-linker such as di isocyanates, diaryl carbonates, dimethly carbonate, diphenyl carbonate, and carbonyl diimidazoles, carboxylic acid dianhydrides. The surface charge density, porosity, and pore sizes of sponges can be able to controlled to attach different molecules. NS which has low cross-linking gives a fast drug release. ${ }^{[9]}$

\section{Emulsion solvent diffusion method}

In this method different proportion of ethyl cellulose and also polyvinyl alcohol is used. The dispersed phase which contains ethyl cellulose and drug were dissolved in $20 \mathrm{ml}$ dichloromethane and slowly added it to a definite amount of polyvinyl alcohol in $150 \mathrm{ml}$ of the aqueous continuous phase. The above reaction mixture was stirred at $1000 \mathrm{rpm}$ for $2 \mathrm{~h}$. Then, NS formed was collected by the filtration and was dried in the oven at $400^{\circ} \mathrm{C}$ for $24 \mathrm{~h}$. The dried NS was stored in the vacuum desiccators to ensure the removal of residual solvent. ${ }^{[10]}$

\section{Ultrasound-assisted synthesis}

In this method, NSs were obtained by reacting polymers with the cross-linkers in the absence of solvent and under the sonication. The NSs obtained using this method will be spherical and uniform in the size. ${ }^{[1]}$ The polymer was mixed and the cross-linker in a particular molar ratio in the flask. The flask was placed in an ultrasound bath which was filled with water and heated it to $90^{\circ} \mathrm{C}$. This mixture was sonicated for $5 \mathrm{~h}$. Then, the mixture was cooled, and the product was broken roughly. The product was washed with water to remove the nonreacted polymer and it was subsequently purified by prolonged Soxhlet extraction with the ethanol. The obtained product was dried under the vacuum and was stored at $25^{\circ} \mathrm{C}$ until further use. ${ }^{[11,12]}$

\section{Loading of drug into NSs}

NSs for drug delivery should be pretreated to obtain a mean particle size below $500 \mathrm{~nm}$. The NSs were suspended in water and sonicated to avoid the presence of aggregates and then centrifuged the suspension to obtain the colloidal fraction. The supernatant was separated and dried the sample by freeze drying. ${ }^{[12]}$ The aqueous suspension of NSs was prepared and 
dispersed the excess amount of the drug and maintained the suspension under constant stirring for the specific time required for complexation. After complexation, the uncomplexed (undissolved) drug was separated from complexed drug by centrifugation. Then, the solid crystals of NSs were obtained by solvent evaporation or by freeze drying. ${ }^{[12,13]}$ Crystal structure of NS plays a very important role in complexation with drug. A study revealed that paracrystalline NSs showed different loading capacities when compared to crystalline NSs. The drug loading is greater in crystalline NSs than paracrystalline one. In poorly crystalline NSs, the drug loading occurs as a mechanical mixture rather than inclusion complex. ${ }^{[13]}$

\section{FACTORS AFFECTING DRUG RELEASE FROM NSs}

1. Physical and chemical properties of entrapped actives

2. Physical properties of sponge system such as pore diameter, pore volume, and resiliency.

3. Properties of vehicle, in which the sponges are finally dispersed

4. Particle size, pore characteristics, and compositions can be considered as imperative parameters

5. External triggers such as pressure, temperature, and solubility of actives

6. Pressure: Pressure or rubbing can release active ingredient from microsponges onto skin

7. Temperature: Some entrapped actives can be too viscous at room temperature to flow spontaneously from sponges onto the skin but increased skin or environment temperature can result in increased flow rate and ultimately drug release ${ }^{[13]}$

8. Solubility: Sponges loaded with water-soluble ingredients such as antiperspirants and antiseptics release the ingredient in the presence of water. ${ }^{[14]}$

\section{CHARACTERIZATION OF NSs}

\section{Microscopy studies}

Scanning electron microscopy and transmission electron microscopy are used to study the morphology and the surface topography of drug, NSs, and the product. The difference in the crystalline state of the raw materials and the product is observed under electron microscope indicates the formation of inclusion complexes. ${ }^{[13]}$ The thermoanalytical methods determine whether the drug substance should undergo some changes before the thermal degradation of the NS. The change of a drug substance may be evaporation, decomposition, melting, oxidation, or the polymorphic transition. The change of drug substance indicates the complex formation. The thermogram which is obtained by differential thermal analysis and differential scanning calorimetry can be observed for broadening, shifting, and appearance of new peaks or disappearance of certain peaks. ${ }^{[15]}$

\section{X-ray diffractometry and single crystal X-ray structure analysis}

The powder X-ray diffractometry can be used to detect the inclusion complex in the solid state. When the drug molecule is in a liquid form since liquid have no diffraction pattern of their own, so the diffraction pattern of a newly formed substance clearly differs from that of uncomplexed NSs. Thus, the difference of diffraction pattern indicates the complex formation. When a drug compound is a solid substance, a comparison has to be made between the diffractogram of the assumed complex and of mechanical mixture of the drug and polymer molecules. ${ }^{[15]}$

A diffraction pattern of a physical mixture is often the sum of those of each component, whereas the diffraction pattern of complexes is apparently different from each of the constituent and leads to a "new" solid phase with the different diffractograms. The diffraction peaks for a mixture of compounds are useful in the determination of the chemical decomposition and complex formation. Complex formation of drug with NSs alters the diffraction patterns and also changes the crystalline nature of the drug. The complex formation sharpens the existing peaks, appearance of a few new peaks and shifting of certain peaks. ${ }^{[15]}$

\section{Single crystal X-ray structure analysis}

This is used to determine the detailed inclusion structure and mode of the interaction. The interaction is between the host and guest molecules and that can be identified and the precise geometrical relationship can also be established. ${ }^{[15]}$

\section{Infra-red spectroscopy}

This method is used to estimate the interaction between NSs and the drug molecules in the solid state. NSs bands often change slightly when complex is formed. If the fraction of guest molecules is encapsulated in the complex is $<25 \%$, bands which could be assigned to include in part of the guest molecules are easily masked by the bands of the spectrum of NSs. This technique is not generally suitable for the detection of the inclusion complexes and is less clarifying than other methods. ${ }^{[15]}$ The application of the infra-red spectroscopy is limited to drugs having some of the characteristic bands, such as sulfonyl or carbonyl groups.

\section{Thin layer chromatography}

In this, the $\mathrm{Rf}$ values of a drug molecule diminish to considerable extent and this helps in identifying the complex formation between the drug and the NS. ${ }^{[15]}$ 


\section{Loading efficiency}

The loading efficiency of NSs can be determined by the quantitative estimation of drug loaded into NSs using UV spectrophotometer and high-performance liquid chromatography method. ${ }^{[16]}$ The loading efficiency (\%) of NSs can be calculated according to the following equation. ${ }^{[17]}$

Actual drug content

Loading efficiency $=\frac{\text { in nanosponge }}{\text { Theoretical drug content }} \times 100$

\section{Solubility studies}

In this, the most widely used approach to study inclusion complexation is the phase solubility method described by Higuchi and Connors, which examines the effect of the NSs on the solubility of the drug. The phase solubility diagrams indicate the degree of complexation. ${ }^{[13-15]}$ In this method, drug was added to the Erlenmeyer flask containing an aqueous solution of various percentages of the NSs. Erlenmeyer flask was stirred on a mechanical shaker at the room temperature. When the steady state was reached, the suspension was filtered by centrifugation process using a 3000 Dalton molecular filter (MICRON YN 30, Millipore Corporation, Bedford MA 1730 U.S.A). The obtained solution was analyzed to determine the drug concentration using high performance liquid chromatography. ${ }^{[18]}$

\section{Particle size and polydispersity}

Particle size is determined by dynamic light scattering using 90 Plus particle size reequipped with MAS OPTION particle sizing software. From this, the mean diameter and polydispersity index can be measured. Measurements were made at the fixed angle of $90^{\circ}$ for all the samples. The samples were suitably diluted with Milli-Q water for every measurement. ${ }^{[16]}$

\section{Zeta potential}

The zeta potential is known as the measure of a surface charge, which is measured using additional electrode in the particle size equipment. And for this type of zeta potential determination, samples of the NSs were diluted with KCL $0.1 \mathrm{~mole} / \mathrm{L}$ and placed in the electrophoretic cell, where the electric field of $15 \mathrm{~V} / \mathrm{cm}$ was applied. Thus, the mean hydrodynamic diameter and the polydispersity index of particles were calculated using the cumulated analysis after averaging of all the total measurements. ${ }^{[19]}$

\section{APPLICATION OF NSs}

Due to their biocompatibility and versatility, NSs have many applications in the field of pharmacy. They can be used as excipients in preparation of tablets, capsules, pellets, granules, suspensions, solid dispersions, or topical dosage forms. They can encapsulate the variety of drugs as shown in Table 1. The NSs can act as a multifunctional carriers for enhanced product performance and elegancy, extended release, irritation is reduced, improved thermal, physical, and the chemical stability of product. Following there are some of the application of NSs which shows versatility of NSs. ${ }^{[20]}$

\section{In solubility enhancement}

NSs improved the solubility of the drug more than 27-fold. When the copolyvidonum was added as a supporting component to the NS formulation, this exceeded to 55 -fold. NSs solubilize drug by possibly masking the hydrophobic groups of itraconazole, by increasing the wetting of the drug, and/or by decreasing the crystallinity of the drug. ${ }^{[19]}$

\section{In drug delivery}

Because of their nanoporous structure, NSs can advantageously carry water-insoluble drugs and/or agents (BCS Class-II drugs). These complexes can be used to increase the dissolution rate, solubility, and stability of drugs, to mask unpleasant flavors and to convert liquid substances to solids. $\beta$-Cyclodextrin-based NSs are reported to deliver the drug to the target site three to five times more effectively than direct injection. ${ }^{[21]}$ Drugs which are particularly critical for formulation in terms of their solubility can be successfully delivered by loading into the NSs. List of some BCS Class II drugs that could be developed as NSs. ${ }^{[22]}$ The NSs are solid

Table 1: Different polymers for nanosponge formulation ${ }^{[22-38]}$

\begin{tabular}{lll} 
Polymers & Copolymers & Cross-linkers \\
\hline Hyper cross-linked polystyrenes & Poly (valerolactone allylvalerolactone) & Carbonyl diimidazoles \\
Cyclodextrins and its derivatives like & Poly (valerolactone-allylvalerolactone & Carboxylic acid dianhydrides \\
Alkyloxycarbonyl cyclodextrins & oxepanedione) & Diarylcarbonates \\
Methyl $\beta$-cyclodextrin, hydroxy & Ethyl cellulose & Dichloromethane \\
propyl & Polyvinyl alcohol & Diisocyanates \\
$\beta$-cyclodextrins & & Diphenyl carbonate, epichloridine, \\
& & Glutaraldehyde \\
& & Pyromellitic anhydride, 2,2-bis (acrylamido) \\
\hline
\end{tabular}


Table 2: Drugs formulated as nanosponges ${ }^{[39-46]}$

\begin{tabular}{|c|c|c|}
\hline Drugs & $\begin{array}{l}\text { Nanosponge } \\
\text { vehicle }\end{array}$ & $\begin{array}{l}\text { Therapeutic } \\
\text { benefit }\end{array}$ \\
\hline $\begin{array}{l}\text { Antisense } \\
\text { oligonucleotides }\end{array}$ & $\begin{array}{l}\text { Sodium alginate } \\
\text { Poly L-lysine }\end{array}$ & $\begin{array}{l}\text { Cancer therapy } \\
\text { Viral infection } \\
\text { Pathologic disorder }\end{array}$ \\
\hline Tamoxifen & $\beta$-cyclodextrin & Breast cancer \\
\hline Resveratrol & $\beta$-cyclodextrin & $\begin{array}{l}\text { Inflammation } \\
\text { Cardiovascular } \\
\text { disease } \\
\text { Dermatitis } \\
\text { Gonorrhea } \\
\text { Fever } \\
\text { Hyperlipidemia }\end{array}$ \\
\hline Paclitaxel & $\beta$-cyclodextrin & Cancer \\
\hline Itraconazole & $\begin{array}{l}\beta \text {-cyclodextrin } \\
\text { Copolyvidonum }\end{array}$ & Antifungal \\
\hline Camptothecin & $\beta$-cyclodextrin & Cancer \\
\hline Dexamethasone & $\beta$-cyclodextrin & Brain tumor \\
\hline $\begin{array}{l}\text { Econazole } \\
\text { nitrate }\end{array}$ & $\begin{array}{l}\text { Ethyl cellulose } \\
\text { Polyvinyl alcohol }\end{array}$ & Antifungal \\
\hline
\end{tabular}

in nature and can be formulated as oral, parenteral, topical, or inhalation dosage forms. For the oral administration, the complexes may be dispersed in a matrix of excipients, diluents, lubricants, and anticaking agents suitable for the preparation of capsules or tablets. ${ }^{[23]}$ For the parenteral administration, the complex may be simply carried in sterile water, saline, or other aqueous solutions. For topical administration, they can be effectively incorporated into topical hydrogel. ${ }^{[24]}$ The NS technology used in formulation of some drugs (Table 2). The earlier work done on Nanosponges is also studied (Table 3).

\section{As a sustained delivery system}

The drug acyclovir is used widely as an antiviral agent due to its efficacy in the treatment of herpes simplex virus infections. ${ }^{[25]}$ Thus, neither the parenteral nor the oral administration of the currently available formulations of drug acyclovir is able to result in suitable concentrations of the agent reaching at target sites. The absorption of acyclovir in the gastrointestinal tract is slow and incomplete; its pharmacokinetics following oral medication has a high variable. The in vitro release profiles of acyclovir from the two types of NSs showed a sustained

\section{Table 3: Earlier work done on nanosponges}

\section{Name of author Study carried}

Moura and Lago Studied catalytic growth of carbon nanotubes and nanofibers on vermiculite to produce floatable hydrophobic "nanosponges" for oil spill remediation ${ }^{[15]}$

Alongi et al. Reported novel flame retardants containing cyclodextrin nanosponges and phosphorous compounds to enhance ethyl vinyl acetate copolymer combustion properties ${ }^{[21]}$

Wong et al. Reported that three-dimensional nanosponges play an important role in the fractionalization of peptides for proteomic applications ${ }^{[18]}$

Lee et al.

Synthesized graphite-nanofiber-supported porous Pt-Ag nanosponges and mesoporous platinum nanosponges as electrocatalysts for the oxygen reduction reaction. ${ }^{[2,29]}$ The precise control of chiral photoreactions or photochirogenesis is one of the most challenging topics in current photochemistry. A supramolecular approach to photochirogenes provides a convenient and also promising tool to facilitate excited-state chirality transfer from chiral host to prochiral substrate

Arkas et al. Reported that nanosponges have the property of encapsulating organic pollutants from water. Ceramic porous filters can be impregnated with these nanosponges resulting in hybrid organic/inorganic filter modules. These hybrid filter modules were tested for the effective purification of water, employing a variety of water pollutants. It has been established that polycyclic aromatic hydrocarbons can be removed very efficiently (more than $95 \%$ ). Representatives of the pollutant group of trihalogen methanes, monoaromatic hydrocarbons, and pesticides (simazine) can also be removed $(>80 \%)^{[7]}$

Liang et al. Developed the pyromellitate-linked cyclodextrin nanosponges, employed for the first time as supramolecular reaction media for sensitizing the enantio differentiating photoisomerization of (Z)-cyclooctene and (Z, Z)-1,3-cyclooctadiene exhibited unique photochirogenesis behavior significantly different from the conventional sensitizer-modified cyclodextrins ${ }^{[36]}$

Alongi et al. Studied the interaction between $\beta$-cyclodextrin nanosponges and two different ultraviolet stabilizers (namely, 2-hydroxy-4(octyloxy)-benzophenone and triphenyl phosphate) in the photooxidation of polypropylene exposed to UV light have been investigated. A significant decrease of the oxidation induction time has been observed in the presence of $\beta-C D$ nanosponges ${ }^{[21]}$

Yang et al. Developed noncytotoxic scaffolds with a nanometer resolution through using silicon substrates as the backbone. This method was merged an optics-based approach with chemical restructuring to modify the surface properties of an IC-compatible material, switching from hydrophilicity to hydrophobicity. Through this nanofabrication-based approach, they synthesized hydrophobic oxidized silicon nanosponges. This study had demonstrated the potential application of using these silicon-based nanopatterns such as influencing cellular behaviors at desired locations with a micro-/nanometer level ${ }^{[37]}$ 
release of the drug from the two types of NSs indicating the encapsulation of acyclovir within the nanostructures. The percentages of acyclovir released from Carb-NSs and NSs after $3 \mathrm{~h}$ in vitro were approximately $22 \%$ and $70 \%$, respectively. Initial burst effect was not observed for either formulation, proved that the drug was not weakly adsorbed onto the NS surfaces. ${ }^{[26]}$

\section{In enzyme immobilization}

The issue of enzyme immobilization is particularly useful for lipases because it improves their stability and modulates properties such as enantioselectivity and reaction rates. ${ }^{[27]}$ As the consequence, demand for new solid supports, suitable for this family of enzymes is constantly growing on. Boscolo et al. reported that the high catalytic performances of Pseudomonas fluorescens lipase adsorbed on a new type of cyclodextrin-based NSs. ${ }^{[28]}$

\section{As a carrier for delivery of gases}

Gases play an important role in medicine for diagnostic or the treatment purposes. The deficiency of more oxygen supply, named hypoxia, is related to various pathologies, from inflammation to that of cancer. So, it is sometime difficult to deliver oxygen gas in appropriate form and dosage in clinical practice. Cavalli et al. developed NSs formulations as oxygen delivery systems for topical application which have the ability to store and to release the oxygen slowly over time. ${ }^{[29]}$

\section{As a protective agent from light or degradation}

Gamma-oryzanol was encapsulated in the formation of NSs, showing a good protection from the photodegradation. $\mathrm{A} \mathrm{O} / \mathrm{W}$ emulsion and a gel were formulated with the gammaoryzanol-loaded NSs. Gamma-oryzanol is a ferulic acid ester mixture, which has recently attracted a great interest as natural antioxidant and usually employed to stabilize the food and pharmaceutical raw materials, moreover as a sunscreen in cosmetics industry. Its application is limited because of its high instability and photodegradation. ${ }^{[30]}$

\section{In anticancer therapy}

NSs carrying anticancer drugs effectively slows the tumor growth, researcher at Vanderbilt University have developed NSs which can be used as delivery system for anticancer drugs to tumors. They claim that this method is three to five times more effective at reducing tumor growth than direct injection of the drugs. The tiny NSs are filled with a drug load and expose at targeting peptide that binds to radiation-induced cell surface receptors on the tumor. When the sponges encounter tumors cells, they stick to the surface and are triggered to release their cargo [Figure 2]. Studies so

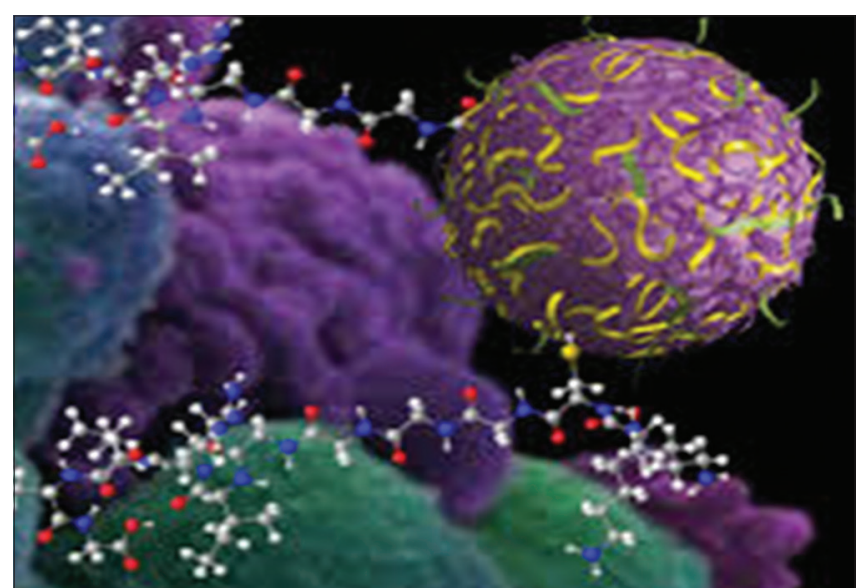

Figure 2: Nanosponges particles attach to a human breast cancer cells

far have been carried out in animals with paclitaxel as the sponge load. The researchers have recorded the response of two different tumors types in animal studies slow-growing human breast cancer and the fast acting mouse glioma-to single injections. The particle holds an anticancer drug that it releases gradually as it decomposes. Peptides linkers are shown with the ball and stick representation, although only two in about three dozen are attached to the surface of actual particles. The linkers are specially configured to bind to the surface of irradiated cancer cells. In both cases, they found that the delivery through NSs increased the death of cancer cells and delayed tumors growth compared with other chemotherapy approaches. ${ }^{[3]}$ Camptothecin, a plant alkaloid and a potent antitumor agent, has a limited therapeutic utility because of its poor aqueous solubility, lactone ring instability, and serious side effects. Cyclodextrins-based NSs are a novel class of cross-linked derivatives of cyclodextrin. They have been used to increase the solubility of poorly soluble actives, to protect the labile groups and control the release. ${ }^{[32]}$

\section{CONCLUSION}

With demand for innovative and highly efficient pharmaceutical and cosmetic products, the market holds considerable potential for NS technology-based formulations and the versatility they offer. As formulators consider new and creative ways to deliver actives, they can realize the full capabilities of these unique assets providing enhanced safety, improved stability, reduced side effects, enhanced multi-functionality, and improved ingredient compatibility. NSs are versatile drug carrier system as they carry both hydrophilic and hydrophobic drugs by forming inclusion and noninclusion complexes. They can deliver drugs by various routes such as oral, topical, and parenteral in a predictable manner to the target site. Besides their application in the drug delivery field, potential applications exist for biomedicine, bioremediation processes, agrochemistry, and catalysis, among others. Drugs delivered by NSs can be proved safe 
and effective, and the pharmaceutical industries will benefit greatly if clinical studies can prove their potential for human use.

\section{REFERENCES}

1. O'Brien JJ, Campoli-Richards DM. Acyclovir. An updated review of its antiviral activity, pharmacokinetic properties and therapeutic efficacy. Drugs 1989;37:233-309.

2. Nacht S, Kantz M. Themicrosponge: Anoveltopical programmable delivery system. In: David WO, Anfon HA, editors. Topical Drug Delivery Systems. Vol. 42. New York: Marcel Dekker; 1992.

3. Davankov VA, Ilyin MM, Tsyurupa MP, Timofeeva GI, Dubrovina LV. From dissolved polystyrene coil to intra molecularly-hyper-cross-linked "nanosponge". Macromolecules 1996;29:8398-403.

4. Khopade AJ, Jain S, Jain NK. The microsponge. East Pharm 1996;25:49-53.

5. Melanie F, Mura P, Adamo M, Maestrelli F, Gratteri P, Bonaccini C. Newdocking CFF91 parameters specific for cyclodextrin inclusion co4mplexes. Chem Phys Lett 2003;370:280-92.

6. Challa R, Ahuja A, Ali J, Khar RK. Cyclodextrins in drug delivery: An updated review. AAPS PharmSciTech 2005;6:E329-57.

7. Arkas M, Allabashi R, Tsiourvas D, Mattausch EM, Perfler R. Organic/inorganic hybrid filters based on dendritic and cyclodextrin "nanosponges" for the removal of organic pollutants from water. Environ Sci Technol 2006;40:2771-7.

8. Trotta F, Cavalli R, Tumiatti W, Zerbinati O, Rogero C, Vallero R. Inventors; Sea Marconi technologies Sas, assignee. Ultrasound assisted synthesis of cyclodextrinbased nanosponges. 2007;1:786-841.

9. Leslie Z, Benet BC. Bioavailability and Bioequivalence. San Francisco, USA: University of California; 2007.

10. Mateo C, Palomo JM, Fernandez-Lorente G, Guisan JM, Fernandez-Lorente R. Improvement of enzyme activity, stabilityand selectivity via immobilization techniques. Enzyme Microb Technol 2007;40:1451-63.

11. Shankar S, Vavia PR, Francesco T, Satyen T. Formulation of betacyclodextrin based nanosponges of itraconazole. J Incl Phenom Macrocycl Chem 2007;57:89-94.

12. Setijadi E, Tao L, Liu J, Jia Z, Boyer C, Davis TP. Biodegradable star polymers functionalized with betacyclodextrin inclusion complexes. Biomacromolecules 2009;10:2699-707.

13. Cavalli R, Rogero CM, Mognetti B, Berta GN, Tumiatti V, Trotta F. Inventors; Sea Marconi Technologies Sas, assignee, Cyclodextrin-based nanosponges as a vehicle for antitumoraldrugs. WO 2009/003656 A1. 2009, January 8 .

14. Eki S, Lei T, Jingquan L, Zhongfan J, Cyrille B, Thomas PD. Biodegradable star polymers functionalized with cyclodextrin inclusion complexes. Biomacromolecule 2009; 10:2699-707.

15. Moura FC, Lago RM. Catalytic growth of carbon nanotubes and nanofibers on vermiculite to produce floatable hydrophobic "nanosponges" for oil spill remediation. Appl Catal B 2009;90:436-40.

16. Singh R, Bharti N, Madan J, Hiremath SN. Characterization of cyclodextrin inclusion complexes-a review. J Pharm Sci Technol 2010;2:171-83.

17. Boscolo B, Trotta F, Ghibaudi E. High catalytic performancesof Pseudomonas fluorescens lipase adsorbed on a new type ofcyclodextrin-based nanosponges. J Mol Catal B Enzym 2010;62:155-61.

18. Wong VN, Fernando G, Wagner AR, Zhang J, Kinsel GR, Zauscher S, et al. Separation of peptides with polyionic nanosponges for MALDI-MS analysis. Langmuir 2009;25:1459-65.

19. Cavalli R, Akhter AK, Bisazza A, Giustetto P, Trotta F, Vavia P. Nanosponge formulations as oxygen delivery systems. Int J Pharm 2010;402:254-7.

20. Swaminathan S, Pastero L, Serpe L, Trotta F, Vavia P, Aquilano D, et al. Cyclodextrin-based nanosponges encapsulating camptothecin: Physicochemical characterization, stability and cytotoxicity. Eur J Pharm Biopharm 2010;74:193-201.

21. Alongi J, Poskovic M, Frache A, Trotta F. Novel flame retardants containing cyclodextrin nanosponges and phosphorous compounds to enhance EVA combustion properties. Polym Degrad Stab 2010;95:2093-100.

22. Arnum PV. Nanosponges, a controlled - Release nnoparticle system, shows promise in targeted drug delivery. Pharm Technol 2011;35:56-60.

23. Sharma R, Walker RB, Pathak K. Evaluation of kinetics and mechanism of drug release from econazole nitrate nanosponge loaded carbopol hydrogel. Indian J Pharm Res Educ 2011;45:25-31.

24. Alongi J, Poskovic M, Frache A, Trotta F. Role of $\beta$-cyclodextrin nanosponges in polypropylene photooxidation. Carbohydr Polym 2011;86:127-35.

25. Lala R, Thorat A, Gargote C. Current trends in $\beta$-cyclodextrin based drug delivery systems. Int J Res Ayurveda Pharm 2011;2:1520-6.

26. Jenny A, Merima P, Alberto F, Francesco T. Role of $\beta$-cyclodextrin nanosponges in propylene photooxidation. Carbohydr Polym 2011;86:127-35.

27. Sharma R, Roderick B, Pathak K. Evaluation and kinetics and hydrogel. Indian J Pharm EduC Res 2011;45:25-31.

28. Lee CL, Chao YJ, Chen CH, Chiou HP, Syu CC. Graphitenanofiber - Supported porous Pt-Ag nanosponges: Synthesis and oxygen reduction electrocatalysis. Int $\mathrm{J}$ Hydrogen Energy 2011;36:15045-51.

29. Lee CL, Wu CC, Chiou HP, Syu CM, Huang CH, Yang CC. Mesoporous platinum nanosponges as electrocatalysts for the oxygen reduction reaction in an acidic electrolyte. Int J Hydrogen Energy 2011;36:6433-40.

30. Rosalba M, Roberta C, Roberto F, Chiara D, Piergiorgio P, 
Leigh E, et al. Antitumor activity of nanosponge Encapsulatedcamptothecin in human prostrate tumors. Cancer Res 2011;71:4431.

31. Sharma R, Pathak K. Polymeric nanosponges as an alternative carrier for improved retention of econazole nitrate onto the skin through topical hydrogel formulation. Pharm Dev Technol 2011;16:367-76.

32. Subramanian S, Singireddy A, Krishnamoorthy K, Rajappan M. Nanosponges: A novel class of drug delivery system - Review. J Pharm Pharm Sci 2012;15:103-11.

33. Patel EK, Oswal RJ. Nanosponge and microsponges: A novel drug delivery system. Int J Res Pharm Chem 2012;2:237-44.

34. Swaminathan S, Vavia PR, Trotta F, Cavalli R, TumbioloS, Bertinetti L, et al. Structural evidence of differentialforms of nanosponges of beta-cyclodextrin and its effect onsolubilization of a model drug. J Incl Phenom Macrocycl Chem 2012;76:201-211.

35. Moya-Ortega MD, Alvarez-Lorenzo C, Concheiro A, Loftsson T. Cyclodextrin-based nanogels for pharmaceutical and biomedical applications. Int J Pharm 2012;428:152-63.

36. Liang W, Yang C, Nishijima M, Fukuhara G, Mori T, Mele A, et al. Cyclodextrin nanosponge-sensitized enantio differentiating photoisomerization of cyclooctene and 1,3-cyclooctadiene. Beilstein J Org Chem 2012;8:1305-11.

37. Yang CY, Liao TC, Shuai HH, Shen TL, Yeh JA, Cheng CM. Micropatterning of mammalian cells on inorganic-based nanosponges. Biomaterials 2012;33:4988-97.

38. Silpa JN, Nissankararao S, Bhimavarapu R, Sravanthi SL, Vinusha K, Renuka K. Nanosponges: A versatile drug delivery system. Int J Pharm Life Sci 2013;4:221-6.

39. Lembo D, Swaminathan S, Donalisio M, Civra A, Pastero L, Aquilano D, et al. Encapsulation of acyclovir in new carboxylated cyclodextrin-based nanosponges improves the agent's antiviral efficacy. Int J Pharm 2013;443:262-72.

40. Sapino S, Carlotti ME, Cavalli R, Ugazio E, Berlier G, Gastaldi L, et al. Photochemical and antioxidant properties of gammaoryzanolin beta-cyclodextrinbased nanosponges. J Incl Phenom Macrocycl Chem 2013;75:69-76.

41. Bolmal UB, Manvi FV, Rajkumar K, Palla SS, Paladugu A, Reddy KR. Recent advances in nanosponges as drug delivery system. Int J Pharm Sci Nanotechnol 2013;6:1934-44.

42. Srinivas P, Sreeja K. Formulation and evaluation of voriconazole loaded nanosponges for oral and topical delivery. Int J Drug Dev Res 2013;5:65-74.

43. Raja CH, Kumar GK, Anusha K. Fabrication and evaluation of ciprofloxacin loaded nanosponges for sustained release. Int J Res Pharm Nano Sci 2013;2:1-9.

44. David F. Nanosponge Drug Delivery System More Effective than Direct Injection. Available from: http:// www.physorg.com. [Last accessed on 2014 Aug 22].

45. Vishwakarma A, Nikam P, Mogal R, Talele S. Review on nanosponges: A benefication for novel drug delivery. Int J PharmTech Res 2014;6:11-20.

46. Tambe RS, Battase PW, Arane PM, Palve SA, Talele SG, Chaudhari G. Review on nanosponges: As a targeted drug delivery system. Am J PharmTech Res 2015;5:215-24.

Source of Support: Nil. Conflict of Interest: None declared. 